\title{
Chiral perturbation theory of muonic-hydrogen Lamb shift: polarizability contribution
}

\author{
Jose Manuel Alarcón ${ }^{1, a}$, Vadim Lensky ${ }^{2,3}$, Vladimir Pascalutsa ${ }^{1}$ \\ ${ }^{1}$ Cluster of Excellence PRISMA Institut für Kernphysik, Johannes Gutenberg-Universität, Mainz 55099, Germany \\ 2 Theoretical Physics Group, School of Physics and Astronomy, University of Manchester, Manchester M13 9PL, UK \\ ${ }^{3}$ Institute for Theoretical and Experimental Physics, Bol'shaya Cheremushkinskaya 25, 117218 Moscow, Russia
}

Received: 6 December 2013 / Accepted: 9 April 2014 / Published online: 29 April 2014

(C) The Author(s) 2014. This article is published with open access at Springerlink.com

\begin{abstract}
The proton polarizability effect in the muonichydrogen Lamb shift comes out as a prediction of baryon chiral perturbation theory at leading order and our calculation yields $\Delta E^{(\mathrm{pol})}(2 P-2 S)=8_{-1}^{+3} \mu \mathrm{eV}$. This result is consistent with most of evaluations based on dispersive sum rules, but it is about a factor of 2 smaller than the recent result obtained in heavy-baryon chiral perturbation theory. We also find that the effect of $\Delta(1232)$-resonance excitation on the Lamb shift is suppressed, as is the entire contribution of the magnetic polarizability; the electric polarizability dominates. Our results reaffirm the point of view that the proton structure effects, beyond the charge radius, are too small to resolve the 'proton radius puzzle'.
\end{abstract}

\section{Introduction}

The eight standard-deviation $(7.9 \sigma)$ discrepancy in the value of proton's charge radius obtained from elastic electronproton scattering [1] and hydrogen spectroscopy [2] on one hand and from the muonic-hydrogen $(\mu \mathrm{H})$ spectroscopy $[3,4]$ on the other, a.k.a. the proton charge radius puzzle $[5,6]$, is yet to meet its fully agreeable solution. One way to solve it is to find an effect that would raise the $\mu H$ Lamb shift by about $310 \mu \mathrm{eV}$, and it has been suggested that proton structure could produce such an effect at $O\left(\alpha_{\mathrm{em}}^{5}\right)$, e.g. [7,8]. Most of the studies, however, derive an order of magnitude smaller effect of proton structure beyond the charge radius [9-15].

The $O\left(\alpha_{\mathrm{em}}^{5}\right)$ effects of proton structure in the Lamb shift are usually divided into the effect of (i) the 3rd Zemach moment, (ii) finite-size recoil, and (iii) polarizabilities. The first two are sometimes combined into (i') the 'elastic' $2 \gamma$ contribution, while the polarizability effect is often split between (ii') the 'inelastic' $2 \gamma$ and (iii') a 'subtraction' term,

a e-mail: alarcon@kph.uni-mainz.de cf. Table 1. The 'elastic' and 'inelastic' $2 \gamma$ contributions are well constrained by the available empirical information on, respectively, the proton form factors and unpolarized structure functions. The 'subtraction' contribution must be modeled, and in principle one can make up a model where the effect is large enough to resolve the puzzle [8].

In this work we observe that chiral perturbation theory $(\chi \mathrm{PT})$ contains definitive predictions for all of the above mentioned $O\left(\alpha_{\mathrm{em}}^{5}\right)$ proton structure effects, hence no modeling is needed, assuming of course that $\chi \mathrm{PT}$ is an adequate theory of the low-energy nucleon structure. Some of the effects were already assessed in the heavy-baryon variant of the theory (HB $\chi$ PT), namely: Nevado and Pineda [11] computed the polarizability effect to leading order (LO) [i.e., $O\left(p^{3}\right)$ ], while Birse and McGovern [13] computed the 'subtraction' term in $O\left(p^{4}\right) \mathrm{HB} \chi \mathrm{PT}$ (with the caveat explained in the end of Sect. 4). Here, on the other hand, we work in the framework of a manifestly Lorentz-invariant variant of $\chi \mathrm{PT}$ in the baryon sector, referred to as $\mathrm{B} \chi \mathrm{PT}$ [16-19]. At least the LO results for nucleon polarizabilities are known to be very different in the two variants of the theory, e.g., the proton magnetic polarizability is (in units of $10^{-4} \mathrm{fm}^{3}$ ): 1.2 in $\mathrm{HB} \chi \mathrm{PT}$ [20] vs. -1.8 in B $\chi$ PT [21-23]. Thus, the LO effect of the pion cloud is paramagnetic in one case and diamagnetic in the other (see [24,25] for more on HB $\chi$ PT vs. B $\chi$ PT). Due to these qualitative and quantitative differences it is interesting to examine the $\mathrm{B} \chi \mathrm{PT}$ predictions for the $2 \gamma$ contributions to the Lamb shift. Here we compute the polarizability effect at LO B $\chi$ PT and indeed find it significantly different from the LO HB $\chi$ PT results of Nevado and Pineda [11]; see Table 1.

Our result for the 'subtraction' and 'inelastic' contributions differ from most of the previous works because we have neglected the effect of the nucleon transition into its lowest excited state-the $\Delta$ (1232). We argue, however (in Sect. 3), that the latter effect cancels out of the polarizability contribution. Thus, even though the 'subtraction' and 'inelastic' 
Table 1 Summary of available calculations of the 'subtraction' (second row), 'inelastic' (third row), and their sum-polarizability (last row) effects on the $2 S$ level of $\mu \mathrm{H}$. The last column represents the $\chi$ PT predictions obtained in this work; here the omitted effect of the $\Delta(1232)$-resonance excitation is missing in the first two ('subtraction' and 'inelastic') numbers, but it does not affect the total polarizability contribution where it is to cancel out

\begin{tabular}{lcclcccc}
\hline$(\mu \mathrm{eV})$ & Pachucki [9] & Martynenko [10] & $\begin{array}{l}\text { Nevado and } \\
\text { Pineda [11] }\end{array}$ & $\begin{array}{l}\text { Carlson and } \\
\text { Vanderhaeghen [12] }\end{array}$ & $\begin{array}{l}\text { Birse and } \\
\text { McGovern [13] }\end{array}$ & $\begin{array}{l}\text { Gorchtein } \\
\text { et al. [14] }\end{array}$ & $\begin{array}{l}\text { LO-B } \chi \text { PT } \\
\text { [this work] }\end{array}$ \\
\hline$\Delta E_{2 S}^{\text {(subt) }}$ & 1.8 & 2.3 & - & $5.3(1.9)$ & $4.2(1.0)$ & $-2.3(4.6)^{\mathrm{a}}$ & -3.0 \\
$\Delta E_{2 S}^{\text {(inel) }}$ & -13.9 & -13.8 & - & $-12.7(5)$ & $-12.7(5)^{\mathrm{b}}$ & $-13.0(6)$ & -5.2 \\
$\Delta E_{2 S}^{\text {(pol) }}$ & $-12(2)$ & -11.5 & -18.5 & $-7.4(2.4)$ & $-8.5(1.1)$ & $-15.3(5.6)$ & $-8.2\left({ }_{-2.5}^{+1.2}\right)$ \\
\hline
\end{tabular}

a Adjusted value; the original value of Ref. [14], +3.3, is based on a different decomposition into the 'elastic' and 'polarizability' contributions

b Taken from Ref. [12]

values appear to be very different from the empirical values due to neglect of the $\Delta(1232)$ excitation, the polarizability contribution is not affected by this neglect.

The details of our calculation and main results are presented in the following section. Remarks on the role of the $\Delta(1232)$ excitation are given in Sect. 3 . The heavy-baryon expansion of our results is discussed in Sect. 4. An "effectiveness" criterion is applied to the $\mathrm{HB} \chi \mathrm{PT}$ and $\mathrm{B} \chi \mathrm{PT}$ results in Sect. 5. The conclusions are given in Sect. 6. Expressions for the LO $\chi$ PT forward doubly virtual proton Compton scattering (VVCS) amplitude and pion electroproduction cross sections are given in Appendices A and B, respectively.

\section{Outline of the calculation and results}

We begin with the leading order chiral Lagrangian for the pion and nucleon fields, as well as the minimally coupled photons; see e.g. [16]. After a chiral rotation of the nucleon field the Lagrangian resembles that of the chiral soliton model; see [26] for details. As the result, the pseudovector $\pi N N$ interaction transforms into the pseudoscalar one, while a new scalar-isoscalar $\pi \pi N N$ interaction is generated. The original and the redefined pion-nucleon Lagrangians, expanded up to the second order in the pion field, take the form

$$
\begin{aligned}
& \mathcal{L}_{\pi N}^{(1)}=\bar{N}\left(i \not \partial-M_{N}+\frac{g_{A}}{2 f_{\pi}} \tau^{a} \not \partial \pi^{a} \gamma_{5}\right. \\
& \left.-\frac{1}{4 f_{\pi}^{2}} \tau^{a} \varepsilon^{a b c} \pi^{b} \not \partial \pi^{c}\right) N+\mathcal{O}\left(\pi^{3}\right), \\
& \mathcal{L}^{\prime}{ }_{\pi N}^{(1)}=\bar{N}\left(i \not \partial-M_{N}-i \frac{g_{A}}{f_{\pi}} M_{N} \tau^{a} \pi^{a} \gamma_{5}\right. \\
& \left.+\frac{g_{A}^{2}}{2 f_{\pi}^{2}} M_{N} \pi^{2}+\frac{\left(g_{A}^{2}-1\right)}{4 f_{\pi}^{2}} \tau^{a} \varepsilon^{a b c} \pi^{b} \not \partial \pi^{c}\right) N+\mathcal{O}\left(\pi^{3}\right),
\end{aligned}
$$

where $N(x)$ and $M_{N}$ is the nucleon field and mass, respectively, $\pi^{a}(x)$ is the pion field; $g_{A} \simeq 1.27, f_{\pi} \simeq 92.4 \mathrm{MeV}$.
Upon the minimal inclusion of the electromagnetic field, the two Lagrangians give identical results for the $O\left(p^{3}\right)$ Compton scattering amplitude and the isovector term proportional to $\left(g_{A}^{2}-1\right)$ does not contribute. Working with the second Lagrangian, however, simplifies a lot the evaluation of the two-loop graphs needed for the Lamb-shift calculation. The resulting Feynman diagrams, omitting crossed and time-reversed ones, are shown in Fig. 1.

These graphs represent an $O\left(\alpha_{\mathrm{em}}^{2}\right)$ correction to the Coulomb potential and can be treated in stationary perturbation theory. Since the Coulomb wave function is $O\left(\alpha_{\mathrm{em}}^{3 / 2}\right)$, the first-order contribution of these graphs to the energy shift is $O\left(\alpha_{\mathrm{em}}^{5}\right)$ as requested. As any energy transfer in the atomic system brings in extra powers of $\alpha_{\mathrm{em}}$, we neglect it, and hence consider strictly the zero-energy forward kinematics. In this case the Feynman amplitude $\mathcal{M}$ is a number in momentum space, corresponding to a potential equal to $\mathcal{M} \delta(\vec{r})$. Because of the $\delta$-function only the $S$-levels are shifted:

$\Delta E_{n S}=\phi_{n}^{2} \mathcal{M}$

where $\phi_{n}^{2}=m_{r}^{3} \alpha_{\mathrm{em}}^{3} /\left(\pi n^{3}\right)$ is the hydrogen wave function at the origin, for $m_{r}=m_{\ell} M_{p} /\left(m_{\ell}+M_{p}\right)$ the reduced mass of the lepton-proton system, and $m_{\ell}, M_{p}=M_{N}$ the corresponding masses of the constituents.

It is customary for the $2 \gamma$ contributions to be split into leptonic and hadronic parts, i.e.,

$\mathcal{M}=\frac{e^{2}}{2 m_{\ell}} \int \frac{\mathrm{d}^{4} q}{i(2 \pi)^{4}} \frac{1}{q^{4}} L_{\mu \nu}(\ell, q) T^{\mu \nu}(P, q)$,

where $e^{2}=4 \pi \alpha_{\mathrm{em}}$ is the lepton charge squared, and

$$
\begin{aligned}
L_{\mu \nu}= & \frac{1}{\frac{1}{4} q^{4}-(\ell \cdot q)^{2}}\left[q^{2} \ell_{\mu} \ell_{\nu}-\left(q_{\mu} \ell_{\nu}+q_{\nu} \ell_{\mu}\right) \ell \cdot q\right. \\
& \left.+g_{\mu \nu}(\ell \cdot q)^{2}\right]
\end{aligned}
$$

is the leptonic tensor, with $\ell$ and $q$ the 4-momenta of the lepton and the photons, respectively; $g_{\mu \nu}=\operatorname{diag}(1,-1,-1$, $-1)$ is the Minkowski metric tensor. The tensor $T^{\mu \nu}$ is the unpolarized VVCS amplitude, which can be written in terms 
Fig. 1 The two-photon exchange diagrams of elastic lepton-nucleon scattering calculated in this work in the zero-energy (threshold) kinematics. Diagrams obtained from these by crossing and time-reversal symmetry are included but not drawn

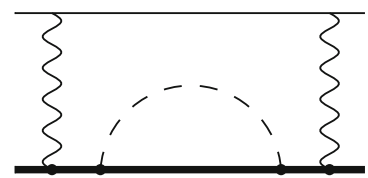

(a)

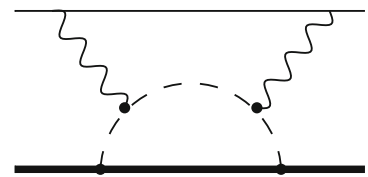

(d)

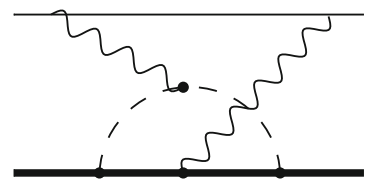

(g)



(b)

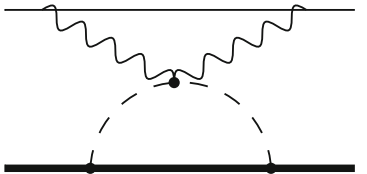

(e)

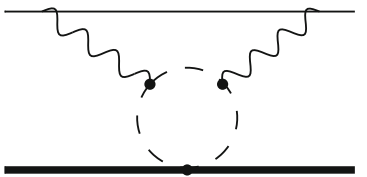

(h)



(c)



(f)



(j) of two scalar amplitudes:

$T^{\mu \nu}(P, q)=-g^{\mu \nu} T_{1}\left(v^{2}, Q^{2}\right)+\frac{P^{\mu} P^{v}}{M_{p}^{2}} T_{2}\left(v^{2}, Q^{2}\right)$,

with $P$ the proton 4-momentum, $v=P \cdot q / M_{p}, Q^{2}=-q^{2}$, $P^{2}=M_{p}^{2}$. Note that the scalar amplitudes $T_{1,2}$ are even functions of both the photon energy $v$ and the virtuality $Q$. Terms proportional to $q^{\mu}$ or $q^{v}$ are omitted because they vanish upon contraction with the lepton tensor.

Going back to the energy shift one obtains [12]:

$$
\begin{aligned}
& \Delta E_{n S}=\frac{\alpha_{\mathrm{em}} \phi_{n}^{2}}{4 \pi^{3} m_{\ell}} \frac{1}{i} \int \mathrm{d}^{3} q \int_{0}^{\infty} \mathrm{d} v \\
& \times \frac{\left(Q^{2}-2 v^{2}\right) T_{1}\left(v^{2}, Q^{2}\right)-\left(Q^{2}+v^{2}\right) T_{2}\left(v^{2}, Q^{2}\right)}{Q^{4}\left[\left(Q^{4} / 4 m_{\ell}^{2}\right)-v^{2}\right]} .
\end{aligned}
$$

In this work we calculate the functions $T_{1}$ and $T_{2}$ by extending the $\mathrm{B} \chi \mathrm{PT}$ calculation of real Compton scattering [26] to the case of virtual photons. We then split the amplitudes into the Born (B) and non-Born (NB) pieces:

$T_{i}=T_{i}^{(\mathrm{B})}+T_{i}^{(\mathrm{NB})}$.

The Born part is defined in terms of the elastic nucleon form factors as in, e.g. $[13,27]$ :

$T_{1}^{(\mathrm{B})}=\frac{4 \pi \alpha_{\mathrm{em}}}{M_{p}}\left[\frac{Q^{4}\left(F_{D}\left(Q^{2}\right)+F_{P}\left(Q^{2}\right)\right)^{2}}{Q^{4}-4 M_{p}^{2} \nu^{2}}-F_{D}^{2}\left(Q^{2}\right)\right]$,

$T_{2}^{(\mathrm{B})}=\frac{16 \pi \alpha_{\mathrm{em}} M_{p} Q^{2}}{Q^{4}-4 M_{p}^{2} v^{2}}\left[F_{D}^{2}\left(Q^{2}\right)+\frac{Q^{2}}{4 M_{p}^{2}} F_{P}^{2}\left(Q^{2}\right)\right]$.

In our calculation the Born part was separated by subtracting the on-shell $\gamma N N$ pion loop vertex in the one-particlereducible VVCS graphs; see diagrams (b) and (c) in Fig. 1.
Focusing on the $O\left(p^{3}\right)$ corrections (i.e., the VVCS amplitude corresponding to the graphs in Fig. 1) we have explicitly verified that the resulting NB amplitudes satisfy the dispersive sum rules [28]:

$$
\begin{aligned}
& T_{1}^{(\mathrm{NB})}\left(v^{2}, Q^{2}\right) \\
& =T_{1}^{(\mathrm{NB})}\left(0, Q^{2}\right)+\frac{2 v^{2}}{\pi} \int_{v_{0}}^{\infty} d v^{\prime} \frac{\sigma_{T}\left(v^{\prime}, Q^{2}\right)}{v^{\prime 2}-v^{2}}, \\
& T_{2}^{(\mathrm{NB})}\left(v^{2}, Q^{2}\right) \\
& =\frac{2}{\pi} \int_{v_{0}}^{\infty} d v^{\prime} \frac{v^{\prime 2} Q^{2}}{v^{\prime 2}+Q^{2}} \frac{\sigma_{T}\left(v^{\prime}, Q^{2}\right)+\sigma_{L}\left(v^{\prime}, Q^{2}\right)}{v^{\prime 2}-v^{2}},
\end{aligned}
$$

with $v_{0}=m_{\pi}+\left(m_{\pi}^{2}+Q^{2}\right) /\left(2 M_{p}\right)$ the pion-production threshold, $m_{\pi}$ the pion mass, and $\sigma_{T(L)}$ the tree-level cross section of pion production off the proton induced by transverse (longitudinal) virtual photons, cf. Appendix B. We hence establish that one is to calculate the 'elastic' contribution from the Born part of the VVCS amplitudes and the 'polarizability' contribution from the non-Born part, in accordance with the procedure advocated by Birse and McGovern [13].

Substituting the $O\left(p^{3}\right)$ NB amplitudes into Eq. (6) we obtain the following value for the polarizability correction:

$\Delta E_{2 S}^{(\mathrm{pol})}=-8.16 \mu \mathrm{eV}$

This is quite different from the corresponding $\mathrm{HB} \chi \mathrm{PT}$ result for this effect obtained by Nevado and Pineda [11]:

$\Delta E_{2 S}^{(\mathrm{pol})}(\mathrm{LO}-\mathrm{HB} \chi \mathrm{PT})=-18.45 \mu \mathrm{eV}$.

We postpone a detailed discussion of this difference till Sect. 4. 
It is useful to observe that a much simpler formulas can be obtained upon making the low-energy expansion (LEX) of the VVCS amplitude, assuming that the photon energy in the atomic system is small compared to all other scales. To leading order in LEX, we may neglect the $v$ dependence in the numerator of Eq. (6) and, after Wick-rotating $q$ to Euclidean hyperspherical coordinates [i.e., setting $v=i Q \cos \chi, \quad \vec{q}=$ $(Q \sin \chi \sin \theta \cos \varphi, Q \sin \chi \sin \theta \sin \varphi, Q \sin \chi \cos \theta)]$ and angular integrations, find the following expression:

$$
\begin{aligned}
\Delta E_{n S}^{(\mathrm{pol})}= & \frac{\alpha_{\mathrm{em}}}{\pi} \phi_{n}^{2} \int_{0}^{\infty} \frac{\mathrm{d} Q}{Q^{2}} w\left(\tau_{\ell}\right) \\
& \times\left[T_{1}^{(\mathrm{NB})}\left(0, Q^{2}\right)-T_{2}^{(\mathrm{NB})}\left(0, Q^{2}\right)\right],
\end{aligned}
$$

with the weighting function $w\left(\tau_{\ell}\right)$ shown in Fig. 2 and given by

$w\left(\tau_{\ell}\right)=\sqrt{1+\tau_{\ell}}-\sqrt{\tau_{\ell}}, \quad \tau_{\ell}=\frac{Q^{2}}{4 m_{\ell}^{2}}$.

Plugging in here the LO B $\chi \mathrm{PT}$ expressions for $T_{i}^{(\mathrm{NB})}$ given in Appendix A, we obtain

$\Delta E_{2 S}^{(\mathrm{pol})}=-8.20 \mu \mathrm{eV}$

i.e., nearly the same as before the LEX, cf. Eq. (10). This comparison shows that the LEX is applicable in this case, i.e.: in the energy-shift formula of Eq. (6) the $v$-dependence of the numerator can to an extremely good approximation be neglected. As shown in Sect. 4, this approximation works well in the case of the $\mathrm{HB} \chi \mathrm{PT}$ calculation too.

To estimate the uncertainty of the LO result, we first observe that for low $Q$ the VVCS amplitudes go as

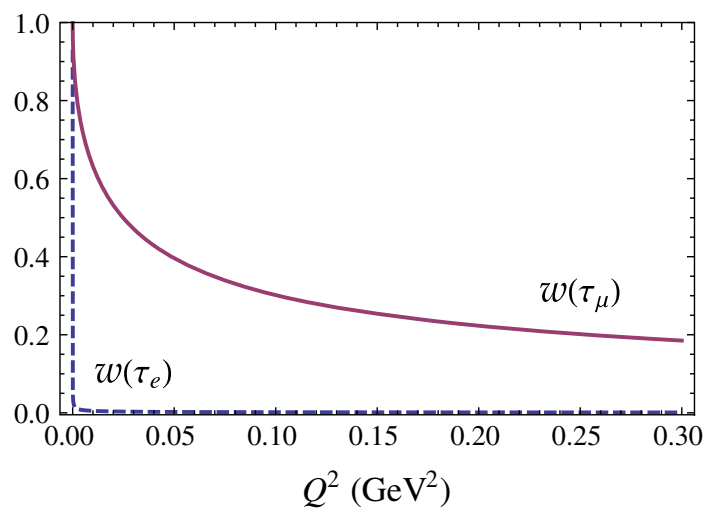

Fig. 2 Plot of the $Q^{2}$ behavior of the weighting function depending on the lepton mass. The blue dashed line is for the case of the electron, $w\left(\tau_{e}\right)$, whereas the solid purple line is for the muon, $w\left(\tau_{\mu}\right)$
$T_{1}^{(\mathrm{NB})}\left(0, Q^{2}\right) \simeq 4 \pi Q^{2} \beta_{M 1}$,

$T_{2}^{(\mathrm{NB})}\left(0, Q^{2}\right) \simeq 4 \pi Q^{2}\left(\alpha_{E 1}+\beta_{M 1}\right)$,

where $\alpha_{E 1}$ and $\beta_{M 1}$ are the electric and magnetic dipole polarizabilities of the proton (hence the name "polarizability contribution"). Given the shape of the weighting function plotted in Fig. 2, the main contribution to the integral in Eq. (12) comes from low $Q$ 's, and therefore $\beta_{M 1}$ cancels out. The dominant polarizability effect in the Lamb shift thus comes from the electric polarizability $\alpha_{E 1}$. The $\mathrm{B} \chi \mathrm{PT}$ physics of $\alpha_{E 1}$ is such that to obtain the empirical number of about 11 (in units of $\left.10^{-4} \mathrm{fm}^{3}\right), 7$ comes from LO $(\pi N$ loops) and 4 from $N L O$ ( $\pi \Delta$ loops), with uncertainty of about \pm 1 from the $O\left(p^{4}\right)$ low-energy constant [26]. Since in the present calculation we include only the LO $\pi N$ loops, we expect our value to increase in magnitude when going to the next order (i.e., including the $\pi \Delta$ loops). As the result, we replace the usual uncertainty of $15 \%\left(\simeq m_{\pi} / \mathrm{GeV}\right)$ due to the higher-order effects by an uncertainty of $30 \%$ $\left[\simeq\left(M_{\Delta}-M_{p}\right) / \mathrm{GeV}\right]$ toward the magnitude increase, anticipating in this way the effect of the $\pi \Delta$ loops. The $15 \%$ uncertainty remains toward the magnitude decrease. With the uncertainty thus defined, our result is

$\Delta E_{2 S}^{(\mathrm{pol})}(\mathrm{LO}-\mathrm{B} \chi \mathrm{PT})=-8.2_{-2.5}^{+1.2} \mu \mathrm{eV}$

This is the number given in the third row of the last column in Table 1, where it can be compared to some previous results. Most of them agree on the polarizability contribution. As for the 'inelastic' and 'subtraction' contributions, their meaningful comparison can only be made together with discussing the role of the $\Delta(1232)$-resonance excitation.

\section{Remarks on the $\Delta(1232)$ contribution and 'subtraction'}

Presently the most common approach to calculate the polarizability effect relies on obtaining the VVCS amplitude from the sum rules of (9). Unfortunately, even a perfect knowledge of the inclusive cross sections (or, equivalently, the unpolarized structure functions) determines the VVCS amplitude only up to the subtraction function $T_{1}^{(\mathrm{NB})}\left(0, Q^{2}\right)$. The total result is therefore divided into the 'inelastic' part which is determined by empirical cross sections, and the 'subtraction' term which stands for the contribution of the subtraction function.

We can also perform such a division and based on the low-energy version of the sum rules [i.e., Eq. (12)] obtain

$$
\Delta E_{n S}^{(\text {subt })}=\frac{\alpha_{\mathrm{em}}}{\pi} \phi_{n}^{2} \int_{0}^{\infty} \frac{\mathrm{d} Q}{Q^{2}} w\left(\tau_{\ell}\right) T_{1}^{(\mathrm{NB})}\left(0, Q^{2}\right) \stackrel{n=2}{=}-3.0 \mu \mathrm{eV},
$$




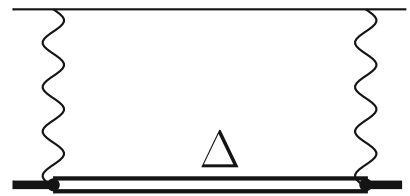

Fig. 3 The $\Delta$ (1232)-excitation mechanism. Double line represents the propagator of the $\Delta$

$$
\Delta E_{n S}^{(\mathrm{inel})}=-\frac{\alpha_{\mathrm{em}}}{\pi} \phi_{n}^{2} \int_{0}^{\infty} \frac{\mathrm{d} Q}{Q^{2}} w\left(\tau_{\ell}\right) T_{2}^{(\mathrm{NB})}\left(0, Q^{2}\right) \stackrel{n \equiv 2}{=}-5.2 \mu \mathrm{eV} .
$$

This looks very different from the dispersive calculation, cf. Table 1 . The main reason for this is the $\Delta(1232)$ resonance excitation mechanism shown by the graph in Fig. 3.

We have checked that the dominant, magnetic-dipole (M1), part of electromagnetic nucleon-to- $\Delta$ transition is strongly suppressed here, as is the entire magnetic polarizability ( $\left.\beta_{M 1}\right)$ contribution, cf. discussion below Eq. (15). It is not suppressed in the 'inelastic' and 'subtraction' contribution separately, but it cancels out in the total. Thus, even though it is well justified to neglect the graph in Fig. 3 at the current level of precision, the split into 'inelastic' and 'subtraction' looks unfair without it.

In most of the dispersive calculations the cancelation of the $\Delta$ excitation, as well as of the entire contribution of $\beta_{M 1}$, occurs too, because the subtraction function is at low $Q$ expressed though the empirical value for $\beta_{M 1}$. Even the $\mathrm{HB} \chi$ PT-inspired calculation of the subtraction function [13], which does not include the $\Delta(1232)$ explicitly, is not an exception, as a low-energy constant from $O\left(p^{4}\right)$ is chosen to achieve the empirical value for $\beta_{M 1}$. Even at $O\left(p^{3}\right)$ $\mathrm{HB} \chi \mathrm{PT}$, the chiral-loop contribution to $\beta_{M 1}$ is-somewhat counterintuitively - paramagnetic and not too far from the empirical value, leading to a reasonable result for the 'subtraction' contribution. We take a closer look at the $\mathrm{HB} \chi \mathrm{PT}$ prediction for the various Lamb-shift contributions in the following section.

The central value for the 'subtraction' contribution obtained by Gorchtein et al. [14] is negative, even though the $\Delta$ excitation is included in their 'inelastic' piece. The quoted uncertainty of their subtraction value, however, is too large to point out any contradiction of this result with the other studies.

\section{Heavy-baryon expansion}

The heavy-baryon expansion, or HB $\chi$ PT [20,29], was called to salvage "consistent power counting" which seemed to be lost in $\mathrm{B} \chi \mathrm{PT}$, i.e. the straightforward, manifestly Lorentz- invariant formulation of $\chi \mathrm{PT}$ in the baryon sector [16]. However, as pointed out by Gegelia et al. [30,31], the "powercounting violating terms" are renormalization scheme dependent and as such do not alter physical quantities. Furthermore, in $\mathrm{HB} \chi \mathrm{PT}$ they are absent only in dimensional regularization. If a cutoff regularization is used the terms which superficially violate power counting arise in $\mathrm{HB} \chi \mathrm{PT}$ as well, and must be handled in the same way as they are handled nowadays in $\mathrm{B} \chi \mathrm{PT}$-by renormalization.

In this work for example, all such (superficially powercounting-violating) terms, together with ultraviolet divergencies, are removed in the course of renormalization of the proton field, charge, anomalous magnetic moment, and mass. We use the physical values for these parameters and hence the on-mass-shell (OMS) scheme. This is different from the extended on-mass-shell scheme (EOMS) [17], where one starts with the parameters in the chiral limit. The physical observables, such as the Lamb shift in this case, would of course come out exactly the same in both schemes, provided the parameters in the EOMS calculation are chosen to yield the physical proton mass at the physical pion mass.

Coming back to $\mathrm{HB} \chi \mathrm{PT}$. Despite the above-mentioned developments the $\mathrm{HB} \chi \mathrm{PT}$ is still often in use. The two EFT studies of proton structure corrections done until now $[11,13]$ are done in fact within $\mathrm{HB} \chi \mathrm{PT}$. We next examine these results from the $\mathrm{B} \chi \mathrm{PT}$ perspective.

One of the advantages of having worked out a $\mathrm{B} \chi \mathrm{PT}$ result is that the one of $\mathrm{HB} \chi \mathrm{PT}$ can easily be recovered. We do it by expanding the expressions of Appendix A in $\mu=m_{\pi} / M_{N}$, while keeping the ratio of light scales $\tau_{\pi}=Q^{2} / 4 m_{\pi}^{2}$ fixed. For the leading term the Feynman-parameter integrations are elementary and we thus obtain the following heavy-baryon expressions:

$$
\begin{aligned}
& T_{1}^{(\mathrm{NB})}\left(0, Q^{2}\right) \stackrel{\mathrm{HB}}{=} \frac{\alpha_{\mathrm{em}} g_{A}^{2}}{4 f_{\pi}^{2}} m_{\pi}\left(1-\frac{1}{\sqrt{\tau_{\pi}}} \arctan \sqrt{\tau_{\pi}}\right), \\
& T_{2}^{(\mathrm{NB})}\left(0, Q^{2}\right) \stackrel{\mathrm{HB}}{=}-\frac{\alpha_{\mathrm{em}} g_{A}^{2}}{4 f_{\pi}^{2}} m_{\pi}\left(1-\frac{1+4 \tau_{\pi}}{\sqrt{\tau_{\pi}}} \arctan \sqrt{\tau_{\pi}}\right) .
\end{aligned}
$$

The first expression reproduces the result of Birse and McGovern (cf. $\bar{T}_{1}^{(3)}$ in the appendix of $[13]^{1}$ ). We have also verified that these amplitudes correspond to the ones

$$
\begin{aligned}
& \overline{1 \text { At subleading order in the heavy-baryon expansion, we obtain }} \\
& \begin{aligned}
\bar{T}_{1}^{\mathrm{NB}(4)} \stackrel{\mathrm{HB}}{=} \frac{\alpha_{\mathrm{em}} g_{A}^{2}}{12 \pi f_{\pi}^{2} M_{N}} & m_{\pi}^{2}\left\{3-50 \tau_{\pi}+\frac{48 \tau_{\pi}\left(1+\tau_{\pi}\right)-3}{\sqrt{\tau_{\pi}\left(1+\tau_{\pi}\right)}} \operatorname{arcsinh} \sqrt{\tau_{\pi}}\right. \\
+ & \left.18 \tau_{\pi}\left[7+4 \log \left(\frac{m_{\pi}}{M_{N}}\right)\right]\right\} .
\end{aligned}
\end{aligned}
$$

This expression reproduces the $g_{A}^{2}$ terms of $\bar{T}_{1}^{(4)}$ in the appendix of Ref. [13], apart from the terms inside the square brackets. These terms 
of Nevado and Pineda [11] at zero energy $(v=0)$, up to a convention for an overall normalization of the amplitudes. We have also reproduced their expressions for $T_{1}$ and $T_{2}$ (cf. Eq. (3.2) and (3.5) in Ref. [11]) for all $v$ and $Q^{2}$.

Substituting these expressions into (12), we obtain the following value for the polarizability contribution to the $2 S$ level shift in $\mu \mathrm{H}$ :

$$
\Delta E_{2 S}^{(\mathrm{pol})}(\mathrm{LO}-\mathrm{HB} \chi \mathrm{PT})=-17.85 \mu \mathrm{eV} .
$$

This is slightly different from the result of Ref. [11] that we quote in Eq. (11), which is because of the neglected energy dependence, i.e., the use of the LEX in deriving Eq. (12) from (6). Still, the difference between the exact and LEX result is well within the expected $15 \%$ uncertainty of such calculation and hence we conclude that the LEX approximation works well in this case too.

Substitution to Eq. (17) yields the $\mathrm{HB} \chi \mathrm{PT}$ predictions for the 'inelastic' and 'subtraction' contributions:

$$
\begin{aligned}
& \Delta E_{2 S}^{\text {(subt) }}(\mathrm{LO}-\mathrm{HB} \chi \mathrm{PT})=1.3 \mu \mathrm{eV}, \\
& \Delta E_{2 S}^{\text {(inel) }}(\mathrm{LO}-\mathrm{HB} \chi \mathrm{PT})=-19.1 \mu \mathrm{eV} .
\end{aligned}
$$

Neglecting for a moment the difference between $\tau_{\pi}$ and $\tau_{\mu}$, we obtain very simple closed expressions for the Lamb-shift contributions:

$$
\begin{aligned}
& \Delta E_{2 S}^{(\mathrm{pol})}(\mathrm{LO}-\mathrm{HB} \chi \mathrm{PT}) \\
& \approx \frac{\alpha_{\mathrm{em}}^{5} m_{r}^{3} g_{A}^{2}}{4\left(4 \pi f_{\pi}\right)^{2}} \frac{m_{\mu}}{m_{\pi}}(1-10 G+6 \log 2)=-16.1 \mu \mathrm{eV}, \\
& \Delta E_{2 S}^{(\mathrm{subt})}(\mathrm{LO}-\mathrm{HB} \chi \mathrm{PT}) \\
& \approx \frac{\alpha_{\mathrm{em}}^{5} m_{r}^{3} g_{A}^{2}}{8\left(4 \pi f_{\pi}\right)^{2}} \frac{m_{\mu}}{m_{\pi}}(1-2 G+2 \log 2)=1.1 \mu \mathrm{eV}, \quad(21 b) \\
& \Delta E_{2 S}^{(\mathrm{inel)})}(\mathrm{LO}-\mathrm{HB} \chi \mathrm{PT}) \\
& \approx \frac{\alpha_{\mathrm{em}}^{5} m_{r}^{3} g_{A}^{2}}{8\left(4 \pi f_{\pi}\right)^{2}} \frac{m_{\mu}}{m_{\pi}}(1-18 G+10 \log 2)=-17.2 \mu \mathrm{eV},
\end{aligned}
$$

where $G \simeq 0.9160$ is the Catalan constant. This should provide an impression of the parametric dependencies arising in $\chi \mathrm{PT}$ for this effect. The resulting numbers are within the expected uncertainty for $\mathrm{HB} \chi \mathrm{PT}$ result, and they can in principle be easily improved in a perturbative treatment of the pion-muon mass difference.

So far we have been discussing the $O\left(p^{3}\right)$ result. At higher orders one in addition to the VVCS calculation needs to consider the appropriate operators from the effective leptonnucleon Lagrangian with corresponding low-energy constants fixed to, e.g., the low-energy lepton-nucleon scatter-

Footnote 1 continued

come from the expansion of the leading pion loop contribution to the term $\beta_{M 1} Q^{2}$ in powers of $m_{\pi}$ and hence are part of $\delta \beta$ in that reference. ing. Birse and McGovern [13] computed the VVCS amplitude $T_{1}\left(0, Q^{2}\right)$ to order $O\left(p^{4}\right)$, but they evaded the consideration of the lepton-nucleon terms by introducing a "physical cutoff" in $Q$. Hence, their resulting calculation of the subtraction term is strongly cutoff dependent and lies, strictly speaking, outside the $\chi \mathrm{PT}$ framework; we refer to it as "HB $\chi \mathrm{PT}$ inspired" calculation.

\section{5 "Effectiveness" of HB $\chi$ PT vs. B $\chi$ PT}

Although at high enough orders $\mathrm{HB} \chi \mathrm{PT}$ and $\mathrm{B} \chi \mathrm{PT}$ are bound to yield the same results, at low orders this is not necessarily so and practice shows that especially at 'predictive' orders, where there are no free low-energy constants to absorb the differences, $\mathrm{HB} \chi \mathrm{PT}$ and $\mathrm{B} \chi \mathrm{PT}$ results differ substantially, sometimes even in the sign of the total effect (cf. the order $p^{3}$ result for the magnetic polarizability of the nucleon $[24,26])$. The proton polarizability contribution to the Lamb shift is apparently such a case as well. So, having found the substantial differences between the $\mathrm{HB} \chi \mathrm{PT}$ and $\mathrm{B} \chi \mathrm{PT}$ predictions the obvious question is: which one is more reliable, if any?

A rather common point of view is that, since $\mathrm{HB} \chi \mathrm{PT}$ neglects only the effects of "higher order", any substantial disagreement only signals the importance of higher-order effects and hence neither of the calculations should be trusted at this order. On the other hand, it is plausible that not all the higher-order effects are large, but only the ones present in the $\mathrm{B} \chi \mathrm{PT}$ calculation and dismissed in the one of $\mathrm{HB} \chi \mathrm{PT}$. In support of the latter scenario is the physical principle of analyticity-consequence of (micro-)causality, which in $\mathrm{B} \chi \mathrm{PT}$ is obeyed exactly, while in $\mathrm{HB} \chi \mathrm{PT}$ it is obeyed only approximately, albeit improvable order by order.

Another, perhaps more quantitative criterion is the one put forward by Strikman and Weiss [32]. In the interpretation of Ref. [24], it requires that the high-momentum contribution of finite (renormalized) loop integrals over quantities which are invariant under redefinitions of hadron fields should not exceed the expected uncertainty of the given-order calculation. In other words, the contribution from beyond the scales at which the effective theory is applicable should not exceed a natural estimate of missing higher-order effects.

In our case the VVCS amplitudes are such quantities invariant under redefinitions of pion and nucleon fields and hence it makes sense to examine Fig. 4, where the polarizability effect is plotted as a function of the ultraviolet cutoff $Q_{\max }$ imposed on the momentum integration in (12).

The figure clearly shows that the relative size of the highmomentum contribution in the $\mathrm{HB} \chi \mathrm{PT}$ case is substantially larger than in $\mathrm{B} \chi \mathrm{PT}$.

Assuming the breakdown scale for $\chi \mathrm{PT}$ is of order of the $\rho$-meson mass, $m_{\rho}=777 \mathrm{MeV}$, we can make a more quanti- 


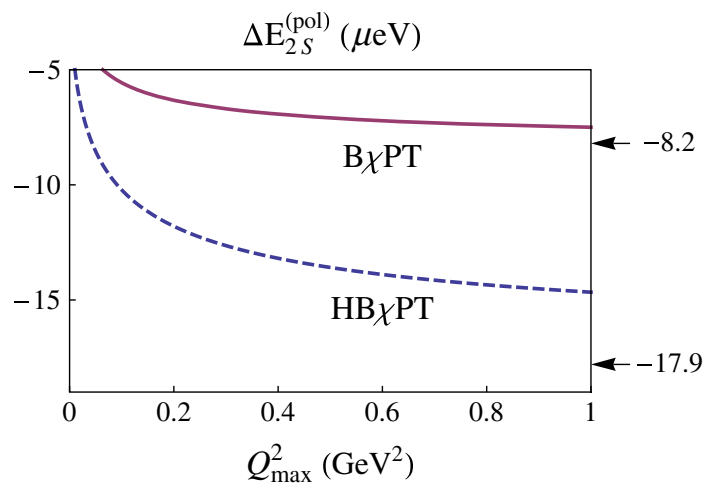

Fig. 4 The polarizability effect on the $2 S$-level shift in $\mu \mathrm{H}$ computed in $\mathrm{HB} \chi \mathrm{PT}$ and $\mathrm{B} \chi \mathrm{PT}$ as a function of the ultraviolet cutoff $Q_{\max }$. The arrows on the right indicate the asymptotic $\left(Q_{\max } \rightarrow \infty\right)$ values

tative statement. In the present $\mathrm{HB} \chi \mathrm{PT}$ calculation the contribution from $Q>m_{\rho}$ is at least $25 \%$ of the total result, hence exceeding the natural expectation of uncertainty of such calculations. In the $\mathrm{B} \chi \mathrm{PT}$ case, the contribution from momenta above $m_{\rho}$ is less than $15 \%$, well within the expected uncertainty.

\section{Conclusion and outlook}

Is the proton polarizability effect different in muonic versus electronic hydrogen so as to affect the charge radius extraction? The answer is 'yes'. From the LEX formula in Eq. (12), one sees that the polarizability contribution not only affects the charge radius extraction from the Lamb shift but also that this effect is about $m_{\mu} / m_{e} \approx 200$ times stronger in $\mu \mathrm{H}$ than in $e \mathrm{H}$. Indeed, the weighting function plotted in Fig. 2 for the two cases is much larger in the muon case. The lepton mass acts, in fact, as a cutoff scale. Nonetheless, the $\mathrm{B} \chi \mathrm{PT}$ result obtained hereby demonstrates that the magnitude of this effect is not nearly enough to explain the 'proton radius puzzle', which amounts to a discrepancy of about $300 \mu \mathrm{eV}$.

As seen from Table 1, our $\mathrm{B} \chi \mathrm{PT}$ result for the polarizability effect agrees with the previous evaluations based on dispersive sum rules, but it is substantially smaller in magnitude than the $\mathrm{HB} \chi \mathrm{PT}$ result of Nevado and Pineda [11]. This is of course not the first case when the $\mathrm{B} \chi \mathrm{PT}$ and $\mathrm{HB} \chi \mathrm{PT}$ results differ significantly-the polarizabilities themselves provide such an example.

The differences between $\mathrm{HB} \chi \mathrm{PT}$ and $\mathrm{B} \chi \mathrm{PT}$ results are often interpreted as the uncertainty of $\chi \mathrm{PT}$ calculations. This interpretation is too naive as there are physical effects that distinguish the two. For example, the $\mathrm{B} \chi \mathrm{PT}$ calculations obey analyticity exactly while the $\mathrm{HB} \chi \mathrm{PT}$ ones only approximately. Furthermore, we have checked that in $\mathrm{HB} \chi \mathrm{PT}$ the contribution from momenta beyond the $\chi \mathrm{PT}$ applicability domain is somewhat bigger than the expected uncertainty of the calculation. The $\mathrm{B} \chi \mathrm{PT}$ result is more "effective" in this respect, as the high-momentum contribution therein is well within the expected uncertainty.

Within the $\mathrm{B} \chi \mathrm{PT}$ calculation, we have verified the dispersive sum rules given in (9) and confirmed the statement of Ref. [13] that the split between the 'elastic' and 'inelastic' $2 \gamma$ contributions corresponds unambiguously to the split between the Born and non-Born parts of the VVCS amplitude, rather than between the pole and non-pole parts.

We have observed that the $\Delta(1232)$-excitation mechanism shown in Fig. 3 does not impact the Lamb shift in a significant way because the dominant magnetic-dipole $(M 1)$ transition is suppressed, as is the entire magnetic polarizability effect. The $\Delta$ (1232)-excitation effect is, however, important for the dispersive calculation because it is prominent in the proton structure functions and hence must be included in the "subtraction' contribution to achieve a consistent cancelation of the $M 1 \Delta(1232)$ excitation. In most of the models this is roughly achieved by using an empirical value for the magnetic polarizability which includes the large paramagnetic effect of the $M 1 \Delta(1232)$ excitation. In the $\mathrm{HB} \chi \mathrm{PT}$-inspired calculation of the 'subtraction' term [13] the $\Delta$-excitation is not included; however, the situation is ameliorated by the low-energy constant from $O\left(p^{4}\right)$, which is chosen to reproduce the empirical value of the magnetic polarizability.

Naive dimensional analysis shows that $\chi \mathrm{PT}$ at leading order is capable of yielding predictions for the entire twophoton correction to the Lamb shift. The polarizability part of that correction has been considered in this work. The last row of the last column of Table 1 contains the $O\left(p^{3}\right) \mathrm{B} \chi \mathrm{PT}$ prediction for the proton polarizability effect on the $2 S$-level of $\mu \mathrm{H}$. One needs to add to it the 'elastic' contribution (or, alternatively, the third Zemach moment together with 'finitesize recoil'), to obtain the full $O\left(\alpha_{\mathrm{em}}^{5}\right)$ effect of the proton structure in $\mu \mathrm{H}$ Lamb shift. Using an empirical value for the 'elastic' contribution from Ref. [13] [i.e., $-24.7(1.6) \mu \mathrm{eV}$ ], our result for the full $2 \gamma$ contribution to the $2 P-2 S$ Lamb shift is in nearly perfect agreement with the presently favored value $[5,13]$ of 33(2) $\mu \mathrm{eV}$.

While the leading-order $\chi \mathrm{PT}$ calculation gives a reliable prediction for the polarizability contribution, the splitting of it into 'inelastic' and 'subtraction' works less well, because of the missing $\Delta$ (1232)-excitation effect, which will only enter at the (future) next-to-leading order calculation. Indeed, $\chi \mathrm{PT}$ is capable of providing results for the Lamb-shift contribution beyond $O\left(p^{3}\right)$. The main difficulty then is to include all the appropriate operators from the effective lepton-nucleon Lagrangian, with corresponding low-energy constants fixed to the two-photon exchange component of the low-energy lepton-nucleon scattering. It will therefore be interesting but very difficult to carry out any beyond-the-leading-order calculation in a systematic way. 
Acknowledgments It is a pleasure to thank M. Birse, C. E. Carlson, M. Gorchtein, R. J. Hill, S. Karshenboim, N. Kivel, J. McGovern, G. A. Miller, A. Pineda, M. Vanderhaeghen, and T. Walcher for insightful, often inspiring, discussions and communications. We furthermore thank A. Antognini, M. Birse, C. E. Carlson, M. Gorchtein, J. McGovern, R. Pohl, M. Vanderhaeghen for helpful remarks on the manuscript. This work was partially supported by the Deutsche Forschungsgemeinschaft (DFG) through the Collaborative Research Center "The LowEnergy Frontier of the Standard Model" (SFB 1044), by the Cluster of Excellence "Precision Physics, Fundamental Interactions and Structure of Matter" (PRISMA), and by the UK Science and Technology Facilities Council through the grant ST/J000159/1. V. L. thanks the Institut für Kernphysik at the Johannes-Gutenberg-Universität Mainz for their kind hospitality.

Open Access This article is distributed under the terms of the Creative Commons Attribution License which permits any use, distribution, and reproduction in any medium, provided the original author(s) and the source are credited.

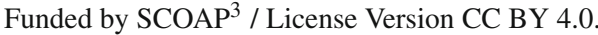

\section{Appendix A: Non-Born amplitudes of zero-energy VVCS}

Here we specify the VVCS amplitudes at $v=0$. The expressions are given in terms of dimensionless variables: the pionproton mass ratio $\mu=m_{\pi} / M_{p}$ and the momentum-transfer $Q$ expressed in the proton mass units. The pre-factor contains the fine-structure constant $\alpha_{\mathrm{em}} \simeq 1 / 137.036$, the proton mass $M_{p} \simeq 938.3 \mathrm{MeV}$, the nucleon axial coupling $g_{A} \simeq 1.27$ and the pion decay constant $f_{\pi} \simeq 92.4 \mathrm{MeV}$. We neglect the isospin breaking effects, such as differences in the nucleon or pion masses. For the latter we assume $m_{\pi} \simeq 139$ $\mathrm{MeV}$.

The $O\left(p^{3}\right) \mathrm{B} \chi \mathrm{PT}$ expressions are given by

$$
\begin{aligned}
& T_{1}^{(\mathrm{NB})}\left(0, Q^{2}\right)=-\frac{\alpha_{\mathrm{em}} g_{A}^{2} M_{p}}{2 \pi f_{\pi}^{2}} \int_{0}^{1} \mathrm{~d} x \\
& \times \int_{0}^{1} \mathrm{~d} y\left\{\sqrt{\frac{4 \mu^{2}}{Q^{2}}+1} \log \left(\frac{\sqrt{\left(4 \mu^{2} / Q^{2}\right)+1}+1}{\sqrt{\left(4 \mu^{2} / Q^{2}\right)+1}-1}\right)\right. \\
& +\frac{3(x-1)}{Q^{2}}\left[\log \left(Q^{2}(-(x-1)) x+\mu^{2} x+(x-1)^{2}\right)\right. \\
& \left.-\log \left(x^{2}+\left(\mu^{2}-2\right) x+1\right)\right] \\
& -\frac{2(x-1)^{2} x\left[(x-1)^{2}\left(Q^{2} y^{2}-1\right)-\mu^{2} x\right]}{\left[(x-1)^{2}\left(Q^{2}(y-1) y-1\right)-\mu^{2} x\right]\left[(x-1)\left(Q^{2}(x-1) y^{2}+Q^{2} y-x+1\right)-\mu^{2} x\right]} \\
& +\frac{(x-1)^{2}(y-1)\left[(x-1)\left(Q^{2}(x-1) y^{2}-Q^{2}(x-2) y+x-1\right)-\mu^{2} x^{2}\right]}{\left[(x-1)\left(Q^{2}(x-1) y^{2}+Q^{2} y-x+1\right)-\mu^{2} x\right]^{2}} \\
& -\frac{4 x^{2}(x-1)(y-1)}{x^{2}\left(Q^{2} y^{2}-1\right)-x\left(\mu^{2}+Q^{2} y-2\right)-1} \\
& -\frac{4 x(x-1)^{2}}{x^{2}\left[Q^{2}(y-1) y-1\right]-\left(\mu^{2}-2\right) x-1} \\
& \left.+\frac{2 x(x-1)}{x^{2}+\left(\mu^{2}-2\right) x+1}-2\right\}, \\
& \quad 22)
\end{aligned}
$$

$$
\begin{aligned}
& T_{2}^{(\mathrm{NB})}\left(0, Q^{2}\right)=-\frac{\alpha_{\mathrm{em}} g_{A}^{2} M_{p}}{\pi f_{\pi}^{2}} \int_{0}^{1} \mathrm{~d} x \int_{0}^{1} \mathrm{~d} y \\
& \quad \times\left\{\frac{(x-1)^{2} x(y-1)\left[(x-1)\left(-Q^{2} y+2 x-2\right)+\mu^{2} x\right]}{\left[(x-1)\left(Q^{2}(x-1) y^{2}+Q^{2} y-x+1\right)-\mu^{2} x\right]^{2}}\right. \\
& +\frac{4(x-1) x^{2} y\left[x^{2}\left(Q^{2}(y-1) y+1\right)-\left(\mu^{2}+2\right) x+1\right]}{\left[x\left(-\mu^{2}+x\left(Q^{2}(y-1) y-1\right)+2\right)-1\right]\left[x^{2}\left(Q^{2} y^{2}-1\right)-x\left(\mu^{2}+Q^{2} y-2\right)-1\right]} \\
& +\frac{4 x}{Q^{2}}\left[\log \left(Q^{2} x y(1-x y)+\mu^{2} x+(x-1)^{2}\right)\right. \\
& \left.-\log \left(x\left(\mu^{2}+x\left(1-Q^{2}(y-1) y\right)-2\right)+1\right)\right] \\
& +\frac{4(x-1) x^{3}(y-1)\left[Q^{2} y(x y-1)-\mu^{2}\right]}{\left[x\left(\mu^{2}+Q^{2}(-x) y^{2}+Q^{2} y+x-2\right)+1\right]^{2}} \\
& +\frac{2(x-1)}{Q^{2}}\left[\frac{(x-1)^{2}\left(Q^{2}(y-1) y+1\right)}{\left[(x-1)^{2}\left(Q^{2}(y-1) y-1\right)-\mu^{2} x\right]}\right. \\
& -\frac{(x-1)^{2}\left(Q^{2}(y-1) y+1\right)}{\left[(x-1)\left(Q^{2}(x-1) y^{2}+Q^{2} y-x+1\right)-\mu^{2} x\right]} \\
& -\log \left[Q^{2}(1-x) y((x-1) y+1)+\mu^{2} x+(x-1)^{2}\right] \\
& \left.+\log \left[\mu^{2} x-(x-1)^{2}\left(Q^{2}(y-1) y-1\right)\right]\right] \\
& -\frac{3}{Q^{4}}\left[-\frac{2 Q^{2} x(x-1)^{2}}{x\left(\mu^{2}+x-2\right)+1}\right. \\
& -\left[\left(Q^{2}-2\right) x+2\right] \log \left[x\left(\mu^{2}+x-2\right)+1\right] \\
& \left.\left.+\left[\left(Q^{2}-2\right) x+2\right] \log \left[x\left(\mu^{2}+Q^{2}(1-x)+x-2\right)+1\right]\right]\right\} .
\end{aligned}
$$

\section{Appendix B: Tree-level electroproduction cross sections}

Here we present our results for the electroproduction cross sections corresponding to diagrams in Fig. 5. We give them in terms of the following dimensionless variables:

$$
\begin{aligned}
& \alpha_{\gamma}=\left(E_{i}^{N}\right)_{\mathrm{cm}} / \sqrt{s}=\frac{s+M_{p}^{2}+Q^{2}}{2 s}, \\
& \alpha_{\pi}=\left(E_{f}^{N}\right)_{\mathrm{cm}} / \sqrt{s}=\frac{s+M_{p}^{2}-m_{\pi}^{2}}{2 s}, \\
& \beta_{\gamma}=E_{\mathrm{cm}}^{\gamma} / \sqrt{s}=\frac{s-M_{p}^{2}-Q^{2}}{2 s}, \\
& \beta_{\pi}=E_{\mathrm{cm}}^{\pi} / \sqrt{s}=\frac{s-M_{p}^{2}+m_{\pi}^{2}}{2 s}, \\
& \lambda_{\gamma}=\left|\vec{q}_{i}\right|_{\mathrm{cm}} / \sqrt{s}=\frac{\sqrt{\left(s-M_{p}^{2}-Q^{2}\right)^{2}+4 s Q^{2}}}{2 s}, \\
& \lambda_{\pi}=\left|\vec{q}_{f}\right|_{\mathrm{cm}} / \sqrt{s}=\frac{\sqrt{\left(s-M_{p}^{2}+m_{\pi}^{2}\right)^{2}-4 s m_{\pi}^{2}}}{2 s},
\end{aligned}
$$

where $\left(E_{i}^{N}\right)_{\mathrm{cm}}$ is the energy of the incoming nucleon, $\left(E_{f}^{N}\right)_{\mathrm{cm}}$ is the energy of the outgoing nucleon, $E_{\mathrm{cm}}^{\gamma}$ the energy of the incoming photon, $E_{\mathrm{cm}}^{\pi}$ the energy of the outgoing pion, $\left|\vec{q}_{i}\right|_{\mathrm{cm}}$ the relative three-momentum of the incoming particles and $\left|\vec{q}_{f}\right|_{\mathrm{cm}}$ the relative three-momentum of the outgoing particles, all in the center-of-mass frame (CM).

We show below the results obtained for the pion electroproduction cross sections for the different channels. They 


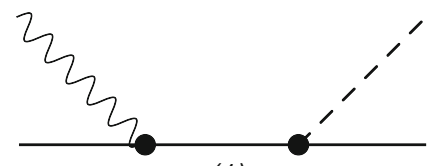

(1)

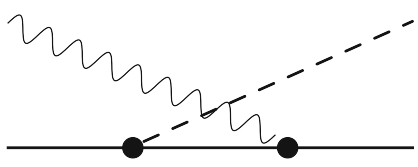

(2)



(3)

Fig. 5 Graphs for pion electroproduction amplitude at leading order. The $\pi N N$ couplings are pseudoscalar as derived from the transformed Lagrangian Eq. (1b)

have been calculated by using the energy of the incoming virtual photons in the laboratory frame as the flux factor of the incoming particles. We have checked that they reproduce the result at the real photon point shown in Refs. [26]. As in Appendix A, $Q$ and $s$ are in the units of proton mass. We have

$$
\begin{aligned}
& \sigma_{T}^{\left(\pi^{+} n\right)}=\frac{\alpha_{\mathrm{em}} g_{A}^{2} \lambda_{\pi}}{4 f_{\pi}^{2} s^{2}\left(s-1+Q^{2}\right) \lambda_{\gamma}^{3}}\left\{\frac { 2 s \lambda _ { \gamma } } { ( s - 1 ) ^ { 2 } } \left[2 \mu ^ { 2 } \left((s-1)^{2}\right.\right.\right. \\
& \left.-Q^{2} s \lambda_{\gamma}^{2}\right)+(1-s)\left(Q^{4}+2 Q^{2} s \beta_{\gamma} \beta_{\pi}\right. \\
& \left.\left.+2 s\left(1-s+2 s \beta_{\gamma} \beta_{\pi}\right) \lambda_{\gamma}^{2}\right)\right] \\
& +\frac{1}{(s-1) \lambda_{\pi}}\left[2 \mu^{2}(1-s)\left(Q^{2}+2 s \beta_{\gamma} \beta_{\pi}\right)\right. \\
& +Q^{2}\left(\left(Q^{2}+2 s \beta_{\gamma} \beta_{\pi}\right)^{2}\right. \\
& \left.\left.\left.-4 s^{2} \lambda_{\pi}^{2} \lambda_{\gamma}^{2}\right)\right] \operatorname{arctanh}\left[\frac{2 s \lambda_{\pi} \lambda_{\gamma}}{Q^{2}+2 s \beta_{\gamma} \beta_{\pi}}\right]\right\} \text {, } \\
& \sigma_{T}^{\left(\pi^{0} p\right)}=\frac{\alpha_{\mathrm{em}} g_{A}^{2} \lambda_{\pi}}{2 f_{\pi}^{2}\left(s-1+Q^{2}\right)(s-1)^{2}} \\
& \times\left\{\frac{1}{-2 s\left(1+s\left(-1+2 \beta_{\gamma} \beta_{\pi}\right)\right)^{2} \lambda_{\gamma}^{2}+8 s^{3} \lambda_{\pi}^{2} \lambda_{\gamma}^{4}}\right. \\
& \times\left[( 1 - s ) \left(Q^{2}\left(s-1-2 s \beta_{\gamma} \beta_{\pi}\right)\right.\right. \\
& \left.-2 s\left(s-1+2 s \beta_{\gamma} \beta_{\pi}\right) \lambda_{\gamma}^{2}\right)\left(\left(1+s\left(-1+2 \beta_{\gamma} \beta_{\pi}\right)\right)^{2}\right. \\
& \left.-4 s^{2} \lambda_{\pi}^{2} \lambda_{\gamma}^{2}\right)+2 \mu^{2}\left(-(s-1)^{2}(1\right. \\
& \left.+s\left(-1+2 \beta_{\gamma} \beta_{\pi}\right)\right)^{2}+2 s\left(Q ^ { 2 } \left(1+2 s\left(-1+\beta_{\gamma} \beta_{\pi}\right)\right.\right. \\
& \left.\left.+s^{2}\left(1+2 \beta_{\gamma} \beta_{\pi}\left(-1+\beta_{\gamma} \beta_{\pi}\right)\right)\right)+2(s-1)^{2} s \lambda_{\pi}^{2}\right) \lambda_{\gamma}^{2} \\
& \left.\left.-4 Q^{2} s^{3} \lambda_{\pi}^{2} \lambda_{\gamma}^{4}\right)\right]+\frac{1}{4 s^{2} \lambda_{\pi} \lambda_{\gamma}^{3}}(1-s) \\
& \times\left[-\left(\left(2 \mu^{2}+Q^{2}\right)(1-s)\right.\right. \\
& \left.+2 Q^{2} s \beta_{\gamma} \beta_{\pi}\right)\left(1+s\left(-1+2 \beta_{\gamma} \beta_{\pi}\right)\right) \\
& \left.+2 s\left(1-2 s+s^{2}+2 Q^{2}\left(\mu^{2}+s \lambda_{\pi}^{2}\right)\right) \lambda_{\gamma}^{2}\right] \\
& \left.\times \operatorname{arctanh}\left[\frac{2 s \lambda_{\pi} \lambda_{\gamma}}{1+s\left(-1+2 \beta_{\gamma} \beta_{\pi}\right)}\right]\right\}, \\
& \sigma_{L}^{\left(\pi^{+} n\right)}=\frac{\alpha_{\mathrm{em}} g_{A}^{2} \lambda_{\pi}}{2 f_{\pi}^{2} Q^{2}\left(s-1+Q^{2}\right)(s-1)^{2} \lambda_{\gamma}^{3}} \\
& \times\left\{\frac{1}{\left(Q^{2}+2 s \beta_{\gamma} \beta_{\pi}\right)^{2}-4 s^{2} \lambda_{\pi}^{2} \lambda_{\gamma}^{2}}\right.
\end{aligned}
$$

$$
\begin{aligned}
& \times\left[2 \lambda _ { \gamma } \left(-Q^{2}(1-s)\left(\beta _ { \gamma } ^ { 2 } \left(Q^{2}\right.\right.\right.\right. \\
& \left.\left.+2 s \beta_{\gamma} \beta_{\pi}\right)+\left(1+s\left(-1+2 \beta_{\gamma}\left(-1+2 \alpha_{\pi}+\beta_{\pi}\right)\right)\right) \lambda_{\gamma}^{2}\right) \\
& \times\left(\left(Q^{2}+2 s \beta_{\gamma} \beta_{\pi}\right)^{2}-4 s^{2} \lambda_{\pi}^{2} \lambda_{\gamma}^{2}\right)+\mu^{2}\left(-2(s-1)^{2}\right. \\
& \times \beta_{\gamma}^{2}\left(Q^{2}+2 s \beta_{\gamma} \beta_{\pi}\right)^{2}+\left(Q^{8}+4 Q^{6} s \beta_{\gamma} \beta_{\pi}\right. \\
& -4 Q^{2}(1-s) s \beta_{\gamma}\left((1-s)\left(-1+\alpha_{\pi}\right)+4 s \beta_{\gamma} \beta_{\pi}\right) \\
& +4 Q^{4} s \beta_{\gamma}\left(s-1+s \beta_{\gamma} \beta_{\pi}^{2}\right) \\
& +4 s^{2}(s-1) \beta_{\gamma}^{2}\left(2 \beta _ { \pi } \left((1-s)\left(-1+\alpha_{\pi}\right)\right.\right. \\
& \left.\left.\left.+2 s \beta_{\gamma} \beta_{\pi}\right)+(s-1) \lambda_{\pi}^{2}\right)\right) \lambda_{\gamma}^{2} \\
& -4 s^{2}\left(\left(1+s^{2}\right)\left(\alpha_{\pi}-1\right)^{2}+\left(Q^{2}+2 s \beta_{\gamma} \beta_{\pi}\right)^{2}\right. \\
& \left.+\left(Q^{4}+4 s^{2} \beta_{\gamma}\right) \lambda_{\pi}^{2}-2 s\left(\left(\alpha_{\pi}-1\right)^{2}+2 \beta_{\gamma} \lambda_{\pi}^{2}\right)\right) \lambda_{\gamma}^{4} \\
& \left.\left.\left.+16 s^{4} \lambda_{\pi}^{2} \lambda_{\gamma}^{6}\right)\right)\right]+\frac{1-s}{s \lambda_{\pi}}\left[\beta_{\gamma}\left(Q^{2}+2 s \beta_{\gamma} \beta_{\pi}\right)\right. \\
& \left.+2 s\left(\alpha_{\pi}-1\right) \lambda_{\pi}^{2}\right]\left[\beta_{\gamma}\left(Q^{4}+2 \mu^{2}(1-s)+2 Q^{2} s \beta_{\gamma} \beta_{\pi}\right)\right. \\
& \left.\left.+2 s\left(2 \mu^{2}+Q^{2} \alpha_{\pi}\right) \lambda_{\gamma}^{2}\right] \operatorname{arctanh}\left[\frac{2 s \lambda_{\pi} \lambda_{\gamma}}{Q^{2}+2 s \beta_{\gamma} \beta_{\pi}}\right]\right\}
\end{aligned}
$$$$
\begin{aligned}
& \sigma_{L}^{\left(\pi^{0} p\right)}=\frac{\alpha_{\mathrm{em}} g_{A}^{2} \lambda_{\pi}}{4 f_{\pi}^{2} Q^{2}\left(s-1+Q^{2}\right)(s-1)^{2} \lambda_{\gamma}^{3}} \\
& \quad \times\left\{\frac { 1 } { ( 1 + s ( - 1 + 2 \beta _ { \gamma } \beta _ { \pi } ) ) ^ { 2 } - 4 s ^ { 2 } \lambda _ { \pi } ^ { 2 } \lambda _ { \gamma } ^ { 2 } } \left[4 \mu ^ { 2 } \lambda _ { \gamma } \left(-(1-s)^{2}\right.\right.\right.
\end{aligned}
$$$$
\times \beta_{\gamma}^{2}\left(1+s\left(-1+2 \beta_{\gamma} \beta_{\pi}\right)\right)^{2}+\left(-2 s\left(1+\alpha_{\pi}\right) \beta_{\gamma}\right.
$$$$
+\left(Q^{4}+2 s^{2} \beta_{\gamma}\left(3+3 \alpha_{\pi}-4 \beta_{\gamma} \beta_{\pi}-2 \alpha_{\pi} \beta_{\gamma} \beta_{\pi}+\beta_{\gamma} \lambda_{\pi}^{2}\right)\right)
$$$$
+s^{2}\left(Q^{4}\left(1+2 \beta_{\gamma} \beta_{\pi}\left(-1+\beta_{\gamma} \beta_{\pi}\right)\right)+2 s^{2} \beta_{\gamma}\left(\alpha_{\pi}-2 \alpha_{\pi} \beta_{\gamma} \beta_{\pi}\right.\right.
$$$$
\left.\left.+\left(1-2 \beta_{\gamma} \beta_{\pi}\right)^{2}+\beta_{\gamma} \lambda_{\pi}^{2}\right)\right)-2 s\left(Q^{4}\left(1-\beta_{\gamma} \beta_{\pi}\right)\right.
$$$$
+s^{2} \beta_{\gamma}\left(3+\alpha_{\pi}\left(3-4 \beta_{\gamma} \beta_{\pi}\right)+2 \beta_{\gamma}\left(2 \beta_{\pi}\left(\beta_{\gamma} \beta_{\pi}-2\right)\right.\right.
$$$$
\left.\left.\left.\left.+\lambda_{\pi}^{2}\right)\right)\right)\right) \lambda_{\gamma}^{2}-2 s^{2}\left(\left(1+\alpha_{\pi}^{2}\right)+Q^{4} \lambda_{\pi}^{2}-2 s\left(1+\alpha_{\pi}^{2}\right.\right.
$$$$
\left.\left.-2 \beta_{\gamma} \beta_{\pi}+2 \beta_{\gamma} \lambda_{\pi}^{2}\right)+s^{2}\left(\alpha_{\pi}^{2}+\left(1-2 \beta_{\gamma} \beta_{\pi}\right)^{2}+4 \beta_{\gamma} \lambda_{\pi}^{2}\right)\right) \lambda_{\gamma}^{4}
$$$$
\left.+8 s^{4} \lambda_{\pi}^{2} \lambda_{\gamma}^{6}\right)-2 Q^{2}(1-s) \lambda_{\gamma}\left(s \beta_{\gamma}^{2}\left(-1+2 \beta_{\gamma} \beta_{\pi}\right)\right.
$$$$
\left.+s\left(1+2 \beta_{\gamma}\left(-2+2 \alpha_{\pi}+\beta_{\pi}\right)\right) \lambda_{\gamma}^{2}+\beta_{\gamma}^{2}-\lambda_{\gamma}^{2}\right)
$$$$
\times\left(1+s\left(-1+2 \beta_{\gamma} \beta_{\pi}-2 \lambda_{\pi} \lambda_{\gamma}\right)\right)
$$$$
\left.\times\left(1+s\left(-1+2 \beta_{\gamma} \beta_{\pi}+2 \lambda_{\pi} \lambda_{\gamma}\right)\right)\right]
$$$$
+\frac{1-s}{s \lambda_{\pi}}\left[2 \mu ^ { 2 } \left(-Q^{4} \lambda_{\gamma}^{2}+\left((1-s) \beta_{\gamma}+2 s \lambda_{\gamma}^{2}\right)\left(\beta_{\gamma}+s \beta_{\gamma}\right.\right.\right.
$$$$
\left.\left.\times\left(-1+2 \beta_{\gamma} \beta_{\pi}\right)+2 s \alpha_{\pi} \lambda_{\gamma}^{2}\right)\right)+Q^{2}\left(\left(\beta_{\gamma}-\lambda_{\gamma}\right)\right.
$$$$
\left.+s\left(\beta_{\gamma}\left(-1+2 \beta_{\gamma} \beta_{\pi}\right)+\lambda_{\gamma}+2\left(\alpha_{\pi}-1\right) \lambda_{\gamma}^{2}\right)\right)\left(\left(\beta_{\gamma}+\lambda_{\gamma}\right)\right.
$$ 


$$
\begin{aligned}
& \left.\left.+s\left(\beta_{\gamma}\left(-1+2 \beta_{\gamma} \beta_{\pi}\right)+\lambda_{\gamma}\left(-1+2\left(\alpha_{\pi}-1\right) \lambda_{\gamma}\right)\right)\right)\right] \\
& \left.\times \operatorname{arctanh}\left[\frac{2 s \lambda_{\pi} \lambda_{\gamma}}{1+s\left(-1+2 \beta_{\gamma} \beta_{\pi}\right)}\right]\right\} .
\end{aligned}
$$

\section{References}

1. J.C. Bernauer et al., [A1 Collaboration], Phys. Rev. Lett. 105, 242001 (2010). arXiv:1307.6227 [nucl-ex]

2. P.J. Mohr, B.N. Taylor, D.B. Newell, Rev. Mod. Phys. 84, 1527 (2012)

3. R. Pohl, A. Antognini, F. Nez, F.D. Amaro, F. Biraben, J.M.R. Cardoso, D.S. Covita, A. Dax et al., Nature 466, 213 (2010)

4. A. Antognini, F. Nez, K. Schuhmann, F.D. Amaro, F. Biraben, J.M.R. Cardoso, D.S. Covita, A. Dax et al., Science 339, 417 (2013)

5. A. Antognini, F. Kottmann, F. Biraben, P. Indelicato, F. Nez, R. Pohl, Ann. Phys. 331, 127 (2013)

6. R. Pohl, R. Gilman, G.A. Miller, K. Pachucki, Ann. Rev. Nucl. Part. Sci. 63, 175 (2013)

7. A. De Rujula, Phys. Lett. B 693, 555 (2010)

8. G.A. Miller, Phys. Lett. B 718, 1078 (2013)

9. K. Pachucki, Phys. Rev. A 60, 3593 (1999)

10. A.P. Martynenko, Phys. Atom. Nucl. 69, 1309 (2006)

11. D. Nevado, A. Pineda, Phys. Rev. C 77, 035202 (2008)

12. C.E. Carlson, M. Vanderhaeghen, Phys. Rev. A 84, 020102 (2011)

13. M.C. Birse, J.A. McGovern, Eur. Phys. J. A 48, 120 (2012)

14. M. Gorchtein, F.J. Llanes-Estrada, A.P. Szczepaniak, Phys. Rev. A 87, 052501 (2013)
15. R.J. Hill, G. Paz, Phys. Rev. Lett. 107, 160402 (2011)

16. J. Gasser, M.E. Sainio, A. Svarc, Nucl. Phys. B 307, 779 (1988)

17. T. Fuchs, J. Gegelia, G. Japaridze, S. Scherer, Phys. Rev. D 68 , 056005 (2003)

18. V. Pascalutsa, M. Vanderhaeghen, S.N. Yang, Phys. Rept. 437, 125 (2007)

19. J.M. Alarcon, J. Martin Camalich, J.A. Oller, Ann. Phys. 336, 413 (2013)

20. V. Bernard, N. Kaiser, U.-G. Meissner, Int. J. Mod. Phys. E 4, 193 (1995)

21. V. Bernard, N. Kaiser, U.-G. Meißner, Phys. Rev. Lett. 67, 1515 (1991)

22. V. Bernard, N. Kaiser, U.-G. Meißner, Nucl. Phys. B 373, 346 (1992)

23. V. Lensky, V. Pascalutsa, Pisma Zh. Eksp. Teor. Fiz. 89, 127 (2009). [JETP Lett. 89, 108 (2009)]

24. J.M.M. Hall, V. Pascalutsa, Eur. Phys. J. C 72, 2206 (2012)

25. V. Lensky, J.A. McGovern, D.R. Phillips, V. Pascalutsa, Phys. Rev. C 86, 048201 (2012)

26. V. Lensky, V. Pascalutsa, Eur. Phys. J. C 65, 195 (2010)

27. D. Drechsel, B. Pasquini, M. Vanderhaeghen, Phys. Rept. 378, 99 (2003)

28. J. Bernabeu, C. Jarlskog, Nucl. Phys. B 60, 347 (1973)

29. E. Jenkins, A.V. Manohar, Phys. Lett. B 255, 558 (1991)

30. J. Gegelia, G. Japaridze, Phys. Rev. D 60, 114038 (1999)

31. J. Gegelia, G. Japaridze, X.Q. Wang, J. Phys. G 29, 2303 (2003).arXiv:hep-ph/9910260

32. M. Strikman, C. Weiss, Phys. Rev. D 80, 114029 (2009) 